\title{
On the Within-Sibship Variance
}

\author{
A. Gimelfarb \\ Department of Biostatistics, School of Public Health, \\ University of Michigan, Ann Arbor, Michigan 48109
}

Received June 14, 1982

\begin{abstract}
An approximate formula for the expected within-sibship genotypic variance of a polygenic, diallelic, additive character is obtained for arbitrary recombination between the loci affecting the character. The formula is exact. when there is no recombination, or when the recombination is free. It is also shown that, if the value of $\frac{1}{2} V_{k}$ (one-half of the parental genotypic variance) is assigned to the withinsibship genotypic variance, as in the model of Cavalli-Sforza and Feldman (1976, Proc. Natl. Acad. Sci. USA 73, 1689-1692), it implies the assumptions of random mating and of the perfect linkage. If, on the other hand, the value of $\frac{1}{2} V^{0}$ (one-half of the linkage equilibrium genotypic variance) is assigned to the within-sibship variance, as in the model of Rice, Cloninger, and Reich (1978, Amer. J. Hum. Genet. 30, 618-643), it implies the assumptions of random mating and either of the free recombination, or of the linkage equilibrium, if the recombination is not free.
\end{abstract}

A model of the quantitative character dynamics was introduced by Cavalli-Sforza and Feldman (1976), and has been used by them (CavalliSforza and Feldman, 1978; Feldman and Cavalli-Sforza, 1977, 1979, 1981) and by others (Rice et al., 1978; Cloninger et al., 1979) to investigate some aspects of the quantitative character dynamics under genetic and cultural modes of inheritance. With respect to the genetic inheritance, this model may be called "zygotic," since the zygote is treated as the elementary unit of genetic inheritance, unlike classical "polygenic" models of population genetics, where the gene is the elementary unit. A zygote in a zygotic model is characterized not by its genotype, but rather by its genotypic value, i.e., by the total contribution of the genotype to the quantitative character. All the processes of genetic transmission in this model are described in a generalized form by a function $L(z \mid x, y)$, whose meaning is that of the conditional probability for an offspring of parents with genotypic values $x$ and $y$ to have the genotypic value $z$.

It is usually assumed that the distribution $L(z \mid x, y)$ is Gaussian with the first moment, i.e., the within-sibship mean genotypic value, equal to the midparental value:

$$
m_{\mathrm{sib}}(x, y)=\int z L(z \mid x, y) d z=\frac{1}{2}(x+y) .
$$


In terms of the classical polygenic models, this implies that the quantitative character is additive.

It is always assumed that the within-sibship variance of the genotypic values is the same for all sibships in a given generation, independent of the parental genotypic values, i.e.,

$$
v_{\mathrm{sib}}(x, y)=\int z^{2} L(z \mid x, y) d z-m_{\mathrm{sib}}^{2}(x, y)=v_{\mathrm{sib}} .
$$

Two arguments have been suggested to justify this assumption. CavalliSforza and Feldman (1976) argued that it may hold approximately, if the number of loci affecting the character is sufficiently large. Slatkin and Lande (1976) pointed out that substantial deviations from this assumption may be expected only for those sibships where both parents are highly homozygous, i.e., have extreme genotypic values. Since occurrence of sibships of this kind must be rare in natural populations, it is argued that this assumption holds approximately for a natural population. (It should be noted that Slatkin and Lande dealt with a model where the elementary unit of hereditary transmission is the phenotype of an individual. Models of this type were introduced by Slatkin (1970) (also Gimelfarb, 1970), and they have been extensively investigated by Karlin $(1979 a-c)$. In spite of their being slightly different, these "phenotypic" models can be treated for all the purposes of this paper in the same manner as the zygotic models.) Although both of the above arguments justifying assumption (2) are questionable, we shall not discuss them. We concentrate instead on the following problem: Assuming that (2) holds, what particular value should be assigned to $v_{\text {sib }}$ ? Notice that assumption (2) is equivalent to replacing the actual genotypic variance within every sibship in a given generation with the expected over all the sibships variance. Therefore, the problem of assigning a particular value to $v_{\text {sib }}$ in (2) is tantamount to the problem of determining the expected withinsibship genotypic variance $v_{\text {sib }}$ in a given generation.

Rice et al. (1978) suggested one-half of the linkage equilibrium genotypic variance in the population under random mating, $V^{0}$ as the value to be assigned to the within-sibship genotypic variance:

$$
v_{\text {sib }}(x, y)=\frac{1}{2} V^{0} .
$$

The zygotic model with such within-sibship variance yields under assortative mating a unique, globally stable equilibrium for the population variance. Condition (3) implies not only that the within-sibship variance is the same for all sibships, but also that it remains the same in any generation, independent of the evolutionary factors affecting the population dynamics.

Cavalli-Sforza and Feldman (1976) assigned to the within-sibship 
variance a value that they consider as "more reasonable" (Feldman and Cavalli-Sforza, 1981):

$$
v_{\text {sib }}(x, y)=\frac{1}{2} V_{k},
$$

where $V_{k}$ is the genotypic variance in the parental population in generation $k$. It is seen that the within-sibship variance in this model, although being the same for all sibships in a given generation, does not necessarily remain constant across generations. An undesirable feature of the zygotic model with such within-sibship variance is that under any positive assortative mating the population variance does not converge to a finite value, whereas for any negative assortative mating it always converges to zero.

Feldman and Cavalli-Sforza (1981) asserted that (3) is an assumption, albeit implicit, of the classical, polygenic, additive model. This assertion has been challenged by Felsenstein (1981), who has demonstrated that in the case of free recombination, the expected within-sibship genotypic variance of a polygenic, additive character under assortative mating is different from $V^{0} / 2$. (His statement that the within-sibship variance is reduced under assortative mating as compared to $V^{0} / 2$ is inaccurate, since, according to his own formula (16), it may increase, if the assortative mating is negative.)

Feldman and Cavalli-Sforza (1981) also pointed out that conditions (3) and (4) "must be regarded as reflections of different sets of assumptions, neither of whose validity can be decided by polemic or consensus or by appealing to higher authority." They have not, however, presented a discussion of what these assumptions might be, nor have they suggested arguments based on either experimental data or on an analysis of existing genetic models in support of either of the conditions (3) and (4).

In this paper, an expression for the expected within-sibship genotypic variance is obtained for the classical, polygenic, additive model with arbitrary recombination. Assumptions about the polygenic model leading to the expected within-sibship variances as in (3) and (4) are also discussed. The appeal to the polygenic model as a basis for a discussion of zygotic models is justified for at least two reasons. First, it is always desirable to have some connections and overlaps between models of different types. Secondly, and more importantly, in view of the lack of experimental data available at the "macro" level of zygotic models, the "micro" level polygenic models, based on relatively elementary but well-established genetic mechanisms, remain the only basis for making more or less objective inferences about and comparisons between different zygotic and other "macro" models of genetic transmission.

The discussion in this paper is based on the previously introduced (Gimelfarb, 1982) "gametic" model of the quantitative character dynamics. This model serves as a connecting link between polygenic and zygotic models, and is called "gametic" because it deals with the gamete as the 
elementary unit of genetic transmission of quantitative characters. A gamete is characterized in this model not by its genotype (gametotype), but by its gametic effect, which is defined as the contribution of the gametotype to the quantitative character. Accordingly, individuals are characterized by the pair $(\alpha, \beta)$ of the gametic effects of the gametes constituting the individual's genotype. The processes of quantitative character development are accounted for in a generalized form by means of a function $f(\alpha, \beta)$ (developmental function), assigning genotypic values to individuals depending on their genotypes expressed in terms of the gametic effects:

$$
x=f(\alpha, \beta) \text {. }
$$

Here, $x$ is the genotypic value of an individual whose genotype is formed by gametes with gametic effects $\alpha$ and $\beta$. (Latin letters will be used for genotypic values and Greek letters for gametic effects.) All processes of the gametic production (gametogenesis) are accounted for in a generalized form by means of the "gametogenetic function" $H(\lambda \mid \alpha, \beta)$, whose meaning is that of the conditional probability for a gamete with the effect $\lambda$ to be among the gametes produced by an individual whose genotype is formed by gametes with the effects $\alpha$ and $\beta$.

The following polygenic model is adopted for the quantitative character under consideration in the future discussion. The character is controlled by $n$ equivalent diallelic loci. The effect of the loci on the character is additive, i.e., the genotypic value of an individual is the sum of the allelic contributions of all the genes constituting the individual's genotype. The character is sex independent, and mutations are neglected.

The gametic effect of a gamete may be assumed for such polygenic models as the sum of the allelic contributions of all the genes constituting the gametic genotype, with the result that the developmental function is simply the sum of the gametic effects:

$$
x=\alpha+\beta
$$

An expression for the first moment of the gametogenetic function,

$$
m_{H}(\alpha, \beta)=\int \lambda H(\lambda \mid \alpha, \beta) d \lambda
$$

was obtained for such a polygenic model (Gimelfarb, 1982) as

$$
m_{H}(\alpha, \beta)=\frac{1}{2}(\alpha+\beta) .
$$

An expression for the second moment,

$$
m_{H}^{\prime \prime}(\alpha, \beta)=\int \lambda^{2} H(\lambda \mid \alpha, \beta) d \lambda
$$


was obtained as

$$
\begin{aligned}
m_{H}^{\prime \prime}(\alpha, \beta)= & \left(\frac{1}{2}-\frac{n}{n-1} R\right)\left(\alpha^{2}+\beta^{2}\right)+2 R \alpha \beta+\frac{n}{n-1} R\left(\varepsilon_{1}+\varepsilon_{2}\right)(\alpha+\beta) \\
& -\frac{2 n^{2}}{n-1} R \varepsilon_{1} \varepsilon_{2}
\end{aligned}
$$

where $n$ is the number of loci, $\varepsilon_{1}$ and $\varepsilon_{2}$ are the contributions of the alternative alleles (it is customary to assume that $\varepsilon_{1}=0$ and $\varepsilon_{2}=1$ ), and $R$ is a parameter determined by the particularities of crossing-over. In the case of crossing-over with the coefficients of recombination being the same between any two adjacent loci, the parameter $R$ is the following function of the number of loci $n$ and the coefficient of recombination $r$ (Gimelfarb, 1982):

$$
R=\left[1-2 r(1-r n)(n+1)-(1-2 r)^{n+1}\right] / 8 r^{2} n^{2} .
$$

It is seen that $R$ can take values between 0 (with any number of loci, when there is no recombination) and 0.25 (when the number of loci increases to infinity with a nonzero recombination).

Expression (8) for the second moment of the gametogenetic function is exactly true if the population of genotypes is regarded statistically (Gimelfarb, 1982). If the population is regarded in the more traditional way, deterministically, then expression (8) is approximate. In at least two cases, however, (8) is the exact expression, even if the population is regarded deterministically: (i) when there is no recombination between the loci, and (ii) when there is free recombination between all the loci. In the case of no recombination, $R=0$ and

$$
m_{H}^{\prime \prime}(\alpha, \beta)=\frac{1}{2}\left(\alpha^{2}+\beta^{2}\right)
$$

In the case of free recombination, $R=(n-1) / 4 n$ and

$$
m_{H}^{\prime \prime}(\alpha, \beta)=\frac{1}{4}\left(\alpha^{2}+\beta^{2}\right)+\frac{n-1}{2 n} \alpha \beta+\frac{1}{4}\left(\varepsilon_{1}+\varepsilon_{2}\right)(\alpha+\beta)-\frac{n}{2} \varepsilon_{1} \varepsilon_{2} .
$$

Thus, (10) and (11) are the exact expressions for the second moment of the gametogenetic function for the polygenic, diallelic, additive model, independent of whether the population of genotypes is regarded statistically or deterministically, in the cases of no recombination and the free recombination respectively. In other cases, (8) provides an approximate expression for the second moment of the gametogenetic function, if the population of genotypes is regarded deterministically. 
In the framework of the gametic model, the expected within-sibship genotypic variance can be computed as

$$
\bar{v}_{\mathrm{sib}}=\int v_{\mathrm{sib}}(\alpha, \beta ; \gamma, \delta) P_{k}(\alpha, \beta ; \gamma, \delta) d(\alpha \beta \gamma \delta)
$$

where $v_{\text {sib }}(\alpha, \beta ; \gamma, \delta)$ is the genotypic variance within a sibship with the parents whose genotypes in the terms of the gametic effects are $(\alpha, \beta)$ and $(\gamma, \delta) . P_{k}(\alpha, \beta ; \gamma, \delta)$ is the distribution of parental pairs in generation $k$. The integration in (12) is over all four variables: $\alpha, \beta, \gamma, \delta$. The variance $v_{\text {sib }}(\alpha, \beta ; \gamma, \delta)$ is computed as

$$
\begin{aligned}
v_{\mathrm{sib}}(\alpha, \beta ; \gamma, \delta)= & \int(\lambda+\eta)^{2} H(\lambda \mid \alpha, \beta) H(\eta \mid \gamma, \delta) d(\lambda \eta) \\
& -m_{\mathrm{sib}}^{2}(\alpha, \beta ; \gamma, \delta),
\end{aligned}
$$

where $m_{\text {sib }}(\alpha, \beta ; \gamma, \delta)$ is the mean within-sibship genotypic value:

$$
m_{\mathrm{sib}}(\alpha, \beta ; \gamma, \delta)=\int(\lambda+\eta) H(\lambda \mid \alpha, \beta) H(\eta \mid \gamma, \delta) d(\lambda \eta) .
$$

Combining (13) and (14) yields after some transformations

$$
v_{\mathrm{sib}}(\alpha, \beta ; \gamma, \delta)=m_{H}^{\prime \prime}(\alpha, \beta)+m_{H}^{\prime \prime}(\gamma, \delta)-m_{H}^{2}(\alpha, \beta)-m_{H}^{2}(\gamma, \delta),
$$

where $m_{H}$ and $m_{H}^{\prime \prime}$ are the first and second moments of the gametogenetic function given in (7) and (8). The expression for the expected within-sibship genotypic variance is obtained by combining (12), (15) and (7), (8):

$$
\begin{aligned}
\bar{v}_{\mathrm{sib}}= & \left(\frac{1}{2}-\frac{2 n R}{n-1}\right) V_{k}+4\left(\frac{2 n-1}{n-1} R-\frac{1}{2}\right) \operatorname{cov}_{k}-\frac{R}{n-1} M_{k}^{2} \\
& +\frac{2 n R}{n-1}\left(\varepsilon_{1}+\varepsilon_{2}\right) M_{k}-\frac{4 n^{2}}{n-1} \varepsilon_{1} \varepsilon_{2} R,
\end{aligned}
$$

where $M_{k}$ and $V_{k}$ are the mean genotypic value and the genotypic variance in the parental population in generation $k$, and $\operatorname{cov}_{k}$ is the covariance of the gametic effects of the gametes forming parental zygotes in generation $k$ :

$$
\operatorname{cov}_{k}=\int \alpha \beta P_{k}(\alpha, \beta) d(\alpha \beta)-\frac{1}{4} M_{k}^{2} .
$$

We shall assume that $M_{k}=M$, i.e., the mean genotypic value remains the same in any generation. It is true, of course, for any additive character in the absence of selection, whereas in presence of selection, this implies that the 
selection does not affect the population mean. It can be proven that under this assumption,

$$
\operatorname{cov}_{k}=\frac{1}{4} \operatorname{Cov}_{k-1}
$$

for any additive character, $\operatorname{Cov}_{k-1}$ being the covariance of the genotypic values of the individuals in parental pairs in generation $k-1$ :

$$
\operatorname{Cov}_{k-1}=\int x y P_{k-1}(x, y) d(x y)-M^{2} \text {. }
$$

Indeed,

$$
\operatorname{cov}_{k}=\int \lambda \eta P_{k}(\lambda, \eta) d(\lambda \eta)-\frac{1}{4} M^{2}
$$

or, using (7),

$$
\operatorname{cov}_{k}=\frac{1}{4} \int(\alpha+\beta)(\gamma+\delta) P_{k-1}(\alpha, \beta ; \gamma, \delta) d(\alpha \beta \gamma \delta)-\frac{1}{4} M^{2},
$$

which yields (18), since for additive characters,

$$
\int x y P_{k-1}(x, y) d(x y)=\int(\alpha+\beta)(\gamma+\delta) \dot{P}_{k-1}(\alpha, \beta ; \gamma, \delta) d(\alpha \beta \gamma \delta) .
$$

The substitution of (18) into (16) produces

$$
\begin{aligned}
\bar{v}_{\mathrm{sib}}= & \left(\frac{1}{2}-\frac{2 n R}{n-1}\right) V_{k}+\left(\frac{2 n-1}{n-1} R-\frac{1}{2}\right) \rho_{k-1} V_{k-1}-\frac{R}{n-1} M^{2} \\
& +\frac{2 n R}{n-1}\left(\varepsilon_{1}+\varepsilon_{2}\right) M \quad \frac{4 n^{2}}{n-1} \varepsilon_{1} \varepsilon_{2} R,
\end{aligned}
$$

where $\rho_{k-1}$ is the genotypic "marital correlation" in generation $k-1$ :

$$
\rho_{k-1}=\operatorname{Cov}_{k-1} / V_{k-1} \text {. }
$$

Expression (20) represents the general formula for the expected withinsibship genetic variance following from the gametic model of a polygenic, diallelic, additive character.

When $R=0$, i.e., in the case of zero recombination (including $n=1$ ), (20) reduces to

$$
\bar{v}_{\text {sib }}=\frac{1}{2} V_{k}-\frac{1}{2} \rho_{k-1} V_{k-1} \text {. }
$$


It has been mentioned earlier that the expression for the second moment of the gametogenetic function (10) is exact in this case. Therefore, $(21)$ is the exact formula for the expected within-sibship genetic variance, if the loci affecting the character are perfectly linked. Under random mating, i.e., when $\rho_{k-1}=0,(21)$ yields (4):

$$
\bar{v}_{\text {sib }}=\frac{1}{2} V_{k},
$$

i.e., the value assigned to the within-sibship variance in the model of CavalliSforza and Feldman.

It is well known that with any amount of recombination (i.e., when $R \neq 0$ ), the genetic variance of a polygenic, diallelic, additive character in a population under random mating attains the linkage equilibrium value $V^{0}$ for which the following formula can be written $((91)$ in Gimelfarb, 1982):

$$
V^{0}=-(1 / 2 n) M^{2}+\left(\varepsilon_{1}+\varepsilon_{2}\right) M-2 n \varepsilon_{1} \varepsilon_{2} .
$$

Taking this into consideration, the following expression for the expected within-sibship genotypic variance is obtained from (20) in the case of nonzero recombination:

$$
\bar{v}_{\mathrm{sib}}=\left(\frac{1}{2}-\frac{2 n R}{n-1}\right) V_{k}+\left(\frac{2 n-1}{n-1} R-\frac{1}{2}\right) \rho_{k-1} V_{k-1}+\frac{2 n R}{n-1} V^{0} .
$$

In the case of free recombination between the loci, $R=(n-1) / 4 n$ and (23) yields

$$
\bar{v}_{\text {sib }}=\frac{1}{2} V^{0}-(1 / 4 n) \rho_{k-1} V_{k-1} .
$$

Since in this case the expression for the second moment of the gametogenetic function (11) is exact, (24) is the exact formula for the within-sibship genotypic variance of a polygenic, diallelic, additive character with the free recombination between all the loci. It can be shown that (24) is equivalent to Felsenstein's (1981) formula (16). Indeed, the second term on the right-hand side of (24) can be represented as

$$
\frac{1}{4 n} \operatorname{Cov}_{k-1}=\frac{\operatorname{cov}_{k}}{n}=\frac{n^{2} \operatorname{cov}_{k}^{*}}{n}=n \operatorname{cov}_{k}^{*},
$$

where $\operatorname{cov}_{k}^{*}$ is the covariance of a gene pair within a zygote in generation $k$, for which the following expression can be written in the Felsenstein's notation:

$$
\operatorname{cov}_{k}^{*}=f_{k} v^{0},
$$

with $v^{0}=2 p q \alpha^{2}$ being the variance within an individual locus. Since $V^{0}$ in 
(24) is the linkage equilibrium genotypic variance, $V^{0}=n v^{0}$, and the Felsenstein formula ensues:

$$
\bar{v}_{\text {sib }}=\frac{1}{2} V^{0}\left(1-f_{k}\right) \text {. }
$$

Felsenstein (1981) concluded from this formula that with the free recombination, the expected within-sibship genotypic variance remains approximately constant, being altered slightly by a fraction of order $1 / n$. Formula (24) indicates, however, that this conclusion is not absolutely correct, since under strong assortative mating, $V_{k}$ may attain values of order $n$ (Crow and Felsenstein, 1968).

Under random mating, i.e., when $\rho_{k-1}=0$, (24) yields (3):

$$
\tilde{v}_{\text {sib }}=\frac{1}{2} V^{0},
$$

i.e., the value assigned to the within-sibship genotypic variance in the model of Rice et al. Notice also that if a population is at equilibrium under random mating, i.e., $p_{k-1}=0$ and $V_{k-1}=V_{k}=V^{0}$, then (23) yields (3):

$$
\bar{v}_{\text {sib }}=\left(\frac{1}{2}-\frac{2 n R}{n-1}\right) V^{0}+\frac{2 n R}{n-1} V^{0}=\frac{1}{2} V^{0}
$$

with any recombination.

The condition $\bar{v}_{\text {sib }}=\frac{1}{2} V_{k}$, when substituted into (23), implies the equality

$$
-\frac{2 n R}{n-1} V_{k}+\left(\frac{2 n-1}{n-1} R-\frac{1}{2}\right) \rho_{k-1} V_{k-1}+\frac{2 n R}{n-1} V^{0}=0 .
$$

The linkage equilibrium variance $V^{0}$ is determined solely by the initial genotypic distribution in the population and may be different depending on the initial distribution. Equality (26), however, must hold for any initial distribution and, consequently, independently of a particular value of $V^{0}$. For this to be true, it is necessary that $R=0$, i.e., there is no recombination. Then, if we neglect the trivial case of $V_{k-1}=0$, it is also necessary that $\rho_{k-1}=0$, i.e., the mating is random.

If the condition $\bar{v}_{\text {sib }}=\frac{1}{2} V^{0}$ is substituted into (23), it implies the equality

$$
\begin{aligned}
\left(\frac{1}{2}\right. & \left.-\frac{2 n R}{n-1}\right) V_{k}+\left(\frac{2 n-1}{n-1} R-\frac{1}{2}\right) \rho_{k-1} V_{k-1} \\
& +\left(\frac{2 n R}{n-1}-\frac{1}{2}\right) V^{0}=0 .
\end{aligned}
$$

For this equality to hold independently of any particular value of $V^{0}$, it is necessary that $R=(n-1) / 4 n$, i.e., that the recombination is free between all 
the loci. It is also necessary that $p_{k-1}=0$, if we neglect again the trivial case of $V_{k-1}=0$.

In the special case when $V_{k}=V_{k-1}=V^{0} \neq 0$ and, consequently, $\rho_{k-1}=$ $\hat{\rho}=0$, both equalities (26) and (27) hold independently of $R$, i.e., for any recombination.

When mating in a population is not random, formula (23) serves as an approximate expression for the within-sibship variance in cases of arbitrary recombination. For example, if the number of loci, $n=10$ and the coefficient of recombination, $r=0.1$, the value of $R$, as computed from (9), is 0.114 . The substitution of this value of $R$ into (23) produces

$$
\bar{v}_{\text {sib }}=0.246 V_{k}-0.259 \rho_{k-1} V_{k-1}+0.254 V^{0} \text {. }
$$

If $n=50$ and $r=0.02$, then the substitution into (23) of $R$, which in this case is equal to 0.109 , produces

$$
\bar{v}_{\text {sib }}=0.277 V_{k}-0.279 \rho_{k-1} V_{k-1}+0.233 V^{0} .
$$

If a population is at the equilibrium under assortative mating, i.e., $\rho_{k-1}=\hat{\rho}$ and $V_{k-1}=V_{k}=\hat{V}$, then it follows from (23) that

$$
\begin{aligned}
\bar{v}_{\mathrm{sib}}= & V^{0}\left[\left(\frac{1}{2}-\frac{2 n R}{n-1}\right) /\left(1-\hat{\rho}\left(1-\frac{1}{2 n}\right)\right)\right. \\
& \left.+\left(\frac{2 n-1}{n-1} R-\frac{1}{2}\right) \hat{\rho} /\left(1-\hat{\rho}\left(1-\frac{1}{2 n}\right)\right)+\frac{2 n R}{n-1}\right],
\end{aligned}
$$

\section{TABLE I}

Expected Within-Sibship Genotypic Variance at Equilibrium under Different Assortative Matings ${ }^{a}$

\begin{tabular}{cll}
\hline$\hat{\rho}$ & \multicolumn{1}{c}{$\bar{v}_{\text {sib }}$} & \multicolumn{1}{c}{$\bar{v}_{\text {sib }}$} \\
\hline 0.00 & $0.500 V^{0}$ & $0.500 V^{0}$ \\
0.25 & $0.492 V^{0}$ & $0.497 V^{0}$ \\
0.50 & $0.476 V^{0}$ & $0.495 V^{0}$ \\
0.75 & $0.435 V^{0}$ & $0.485 V^{0}$ \\
1.00 & 0.000 & 0.000 \\
& $n=10$ & $n=50$ \\
& $r=0.1$ & $r=0.02$ \\
\hline
\end{tabular}

${ }^{a} \hat{\rho}$, Genetic marital correlation; $n$, number of loci; $r$, coefficient of recombination. 
since under assortative mating (Crow and Felsenstein, 1968)

$$
\hat{V}=V^{0} /\left(1-\hat{\rho}\left(1-\frac{1}{2 n}\right)\right) \text {. }
$$

In Table I, the expected values of the within-sibship genotypic variance at the equilibrium under assortative matings with different genetic marital correlations are computed from (30) for polygenic, additive characters controlled by $n=10$ diallelic loci with the coefficient of recombination, $r=0.1$ (second column), and by $n=50$ loci with $r=0.02$ (third column). It can be noticed from observing this table, that, if the assortative mating is not very strong $(\rho<0.75)$, the equilibrium within-sibship variance is close to $\frac{1}{2} V^{0}$ in all the cases.

\section{CONCLUSIONS}

If the value of $\frac{1}{2} V_{k}$ (one-half of the parental genotypic variance) is assigned to the within-sibship genotypic variance, it implies the assumptions of random mating and of the perfect linkage between the loci affecting the character. If, on the other hand, the value of $\frac{1}{2} V^{0}$ (one-half of the linkage equilibrium genotypic variance) is assigned to the within-sibship variance, it implies the assumptions of random mating and either of the free recombination, or of the linkage equilibrium, if the recombination is not free. It can also be concluded, that, although in general the condition $\bar{v}_{\text {sib }}=\frac{1}{2} V^{0}$ does not hold, it may be a very good approximation when the assortative mating is not very strong $(\rho<0.75)$ and the population is close to an equilibrium.

\section{ACKNOWLEDGMENTS}

I am indebted to Dr. Morton Brown for his assistance. I also thank a referee for useful remarks. The work was supported by Public Health Service Grant 5 DO4 AH01744-02.

\section{REFERENCES}

Cavalli-Sforza, L. L., ANd Feldman, M. W. 1976. Evolution of continuous variation: Direct approach through joint distribution of genotypes and phenotypes, Proc. Natl. Acad. Sci. USA 73, 1689-1692.

Cavalli-Sforza, L. L., and Feldman, M. W. 1978. The evolution of continuous variation. III. Joint transmission of genotype, phenotype and environment, Genetics 90, 391-425.

Cloninger, C. R., Rice, J., and Reich, T. 1979. Multifactorial inheritance with cultural transmission and assortative mating. II. A general model of combined polygenic and cultural inheritance, Amer. J. Hum. Genet. 31, 176-198. 
Crow, J. F., And Felsenstein, J. 1968. The effect of assortative mating on the genetic composition of a population, Eugen. Q. 15, 85-97.

Feldman, M. W., and Cavalli-SForza, L. L. 1977. The evolution of continuous variation. II. Complex transmission and assortative mating, Theor. Pop. Biol. 11, 161-181.

Feldman, M. W., and Cavalli-Sforza, L. L. 1979. Aspects of variance and covariance analysis with cultural inheritance, Theor. Pop. Biol. 15, 276-307.

Feldman, M. W., and Cavalli-SForza, L. L. 1981. Assortative mating, selection and mutation models for continuous variation: A reply to Felsenstein, Theor. Pop. Biol. 19, 370-377.

Felsenstein, J. 1981. Continuous genotype models and assortative mating, Theor. Pop. Biol. 19, 341-357.

GimelfarB, A. 1970. The limiting distribution of a character in populations with normal inheritance function, in "Bulletin of Scientific Information on Agronomical Physics," No. 14, Leningrad (in Russian).

Gimelfarb, A. 1982. Quantitative character dynamics: Gametic model, Theor. Pop. Biol. 22 , 324-366.

KARLIN, S. 1979a. Models for multifactorial inheritance. I. Multivariate formulations and basic convergence result, Theor. Pop. Biol. 15, 308-355.

KARLIN, S. 1979b. Models for multifactorial inheritance. 11. The covariance structure for a scalar phenotype under selective assortative mating and sex-dependent symmetric parental transmission, Theor. Pop. Biol. 15, 356-393.

KARLIN, S. $1979 \mathrm{c}$. Models for multifactorial inheritance. II. Calculation of covariance of relatives under extended selective mating mechanisms, Theor. Pop. Biol. 15, 394-423.

Rice, J., Cloninger, C. R., AND REICH, T. 1978. Multifactorial inheritance with cultural transmission and assortative mating. 1. Description and basic properties of the unitary models, Amer. J. Hum. Genet. 30, 618-643.

Slatkin, M. 1970. Selection and polygenic characters. Proc. Natl. Acad. Sci. USA 66, 87-93.

SLATKIN, M., AND LANDE, R. 1976. Niche width in a fluctuating environment-density independent model, Amer. Natur. 119, 341-357. 